\title{
Topical amethocaine gel for pain relief of heel prick blood sampling: a randomised double blind controlled trial
}

A Jain, N Rutter, M Ratnayaka

\begin{abstract}
Background-Heel prick blood sampling is a commonly performed and painful procedure in the newborn infant. Use of a topical local anaesthetic does not relieve this pain. A $4 \% \mathrm{w} / \mathrm{w}$ amethocaine gel (Ametop) reduces the pain of venepuncture in the newborn but has not been tried with heel pricks.
\end{abstract}

Aim-To investigate the effect of topical amethocaine gel on the pain of heel prick in the newborn infant.

Design-Randomised, double blind, placebo controlled trial.

Subjects-Sixty newborn infants, gestation 28-42 weeks (median 36), postnatal age 1-16 days (median 5) undergoing routine heel prick blood sampling.

Methods-A $1.5 \mathrm{~g}$ portion of $4 \% \mathrm{w} / \mathrm{w}$ amethocaine gel or placebo was applied to the skin under occlusion for one hour, then wiped away. Heel prick blood sampling with a spring loaded lance was performed five minutes later. The procedure was videotaped and pain assessed at one second intervals using an adaptation of the neonatal facial coding system (NFCS). No or minimal pain was defined as a cumulative score of less than 5 (out of 15) in the three seconds after firing of the lance and as lack of a cry in the first five seconds.

Results-In terms of a low NFCS core and lack of cry $(p=0.12) 20$ of $30(67 \%)$ in the amethocaine group and 13 of $29(45 \%)$ in the placebo group had no or minimal pain in response to the heel prick. The median cumulative NFCS score over the three seconds after firing the lance was 3 (interquartile range 0-6) in the amethocaine group compared with 5 (interquartile range 1-10) in the placebo group $(p=0.07)$. These differences are not significant.

Conclusions-Topical amethocaine gel does not have a clinically important effect on the pain of heel prick blood sampling and its use for this purpose cannot therefore be recommended. Alternative approaches to the relief of pain from this procedure should be explored.

(Arch Dis Child Fetal Neonatal Ed 2001;84:F56-F59)

Keywords: heel prick; pain; anaesthetic; amethocaine gel

Heel prick blood sampling (HPBS) is the commonest invasive procedure performed on the newborn infant. ${ }^{12}$ It elicits a measurable behavioural response indicating that it is intensely painful for the $b a b y^{3-5}$, more painful than venepuncture. ${ }^{67}$ Multiple heel pricks are performed on some term and many preterm infants with resulting sensitisation rather than tolerance to pain. ${ }^{8}$ There may or may not be longer term effects, ${ }^{9-13}$ but on humane grounds alone such procedural pain should be reduced or avoided where possible. Use of a topical local anaesthetic is one method that has been tried to achieve this. Attempts to relieve the pain of HPBS with topical lignocaine ${ }^{14-16}$ or a lignocaine/prilocaine mixture (EMLA) ${ }^{17} 18$ have been unsuccessful.

Amethocaine is an ester group local anaesthetic available as a topical $4 \%(\mathrm{w} / \mathrm{w})$ gel (Ametop). It has been shown to provide effective and safe local anaesthesia in children for venepuncture and intravenous cannulation. ${ }^{19-21}$ Using the cutaneous withdrawal reflex to assess anaesthesia, we have shown that topical amethocaine gel has a local anaesthetic action in the newborn infant which lasts for up to five hours. $^{22} 23$ In addition, we have shown that it is highly effective in reducing the pain of venepuncture in the term and preterm infant. ${ }^{24}$ In this study, we assess its ability to reduce the pain of HPBS.

\section{Methods}

We conducted a randomised, double blind, placebo controlled trial to investigate the effect of topical amethocaine gel on the pain of HPBS in the newborn infant. We measured the pain response to the use of a spring loaded lance (Autolet; Owen Mumford, UK), the routine method for obtaining blood samples by heel prick on our neonatal unit and postnatal wards. We assessed pain using a validated adaptation of the neonatal facial coding system (NFCS) and by the presence or absence of crying. ${ }^{25}$ The adapted NFCS scores each of the following facial characteristics as present (one point) or absent (no points): eye squeeze, brow bulge, open mouth, deepened nasolabial folds, and cry. We scored the presence or absence of each of these features for each subject. In addition, we recorded the total length of cry in response to HPBS, defined as a cry starting within five seconds of firing the lance and finishing with a gap of at least 30 seconds before any further cry. A Sony CCD-F450E Handycam in long play mode at a tape speed of 0.25 seconds per frame was used to record the heel prick, the facial changes, and the cry. The videotapes were then analysed together at the end of the study. 
Newborn infants having HPBS as part of their routine care were recruited for the study. A $1.5 \mathrm{~g}$ portion of $4 \%(\mathrm{w} / \mathrm{w})$ amethocaine gel or $1.5 \mathrm{~g}$ placebo was applied to the plantar surface of the left or right heel. The placebo was identical in every way except for the absence of the amethocaine component. The gel was covered with an occlusive dressing (Tegaderm) and left alone. After one hour, the occlusive dressing was removed, the excess gel wiped away, and the baby left to settle for five minutes. A midwife, neonatal nurse, or senior house officer performed HPBS. There was no selection of personnel and no specific instructions were given about the way in which the sample should be taken. Blood was taken for routine measurement of haematological or biochemical variables (full blood count, levels of urea and electrolytes, liver function, glucose concentration, bilirubin concentration) or biochemical screening (Guthrie test). The exact moment at which the lance was fired was readily identified on the tape by the noise it made. The period from 20 seconds before firing until the end of the cry was recorded.

Two of the authors (AJ and NR) viewed and scored the videotapes independently. They assigned an NFCS score for each second of a 15 second period, starting five seconds before the heel prick and ending 10 seconds afterwards. The primary outcome measures were defined as: (a) a cumulative NFCS score of less than 5 in the first three seconds after the heel prick (maximum possible score 15) and; (b) the absence of crying at any time within the five second period immediately after firing the lance. Each of these indicating no or minimal pain. These measures were chosen to reflect the pain caused directly by the lance piercing the heel rather than any pain or discomfort caused by squeezing the heel to obtain blood. We also recorded the cumulative NFCS score over the first five and ten seconds after heel prick (maximum possible scores of 25 and 50 respectively) and the total length of the cry. The reason for sampling and the number of attempts needed to obtain the blood sample were noted.

\section{SUBJECTS}

We studied 60 newborn infants from 28 to 42 weeks gestation (median 36) at 1-16 days of age (median 5). They were unselected, but randomisation was within three gestational age groups (term, 33-36 weeks, and 28-32 weeks). Infants admitted to the postnatal wards or Neonatal Unit at Nottingham City Hospital were eligible for entry, but we excluded those who were unwell, ventilated, or sedated. The hospital's research ethics committee approved the study. Infants were studied with the informed written consent of the parent(s). The

Table 1 Details of infants studied

\begin{tabular}{lllll}
\hline Group & $n$ & Postnatal age (days) & Gestation (weeks) & Birth weight $(\mathrm{kg})$ \\
\hline Amethocaine & 30 & $5(1-9)$ & $36.5(28-42)$ & $2.32(1.00-3.94)$ \\
Placebo & 29 & $5(1-16)$ & $36(28-40)$ & $2.40(1.15-3.43)$ \\
p value & & 0.66 & 0.60 & 0.45
\end{tabular}

Values are medians (ranges). p Value obtained by the Mann-Whitney U test.
Medicines Control Agency granted exemption from the restrictions of the product licence of the drug.

\section{RANDOMISATION}

Infants were randomised to have amethocaine or placebo gel applied to the heel. Randomisation was stratified so that half of the subjects in each gestational age group received amethocaine and half received placebo. The gels were packaged in identical tubes by the hospital pharmacy who randomised and coded them. The code was only broken at the end of the study after the videotapes had been scored and when the method of defining a painful or nonpainful response had been agreed on.

\section{STATISTICAL ANALYSIS}

Statistical analysis was performed using SPSS 8.0 software. Tests for normality showed that the data were not normally distributed. The degree of agreement between the assessors in their scoring of the tapes was calculated using the Facial Action Coding System reliability formula $^{26}$ for each of the five characteristics scored at each second of the 15 second measurement period. The proportion of subjects in each group who showed no or minimal painful reaction to the heel prick and the proportion who did not cry were compared using Fisher's exact test. The cumulative NFCS scores over three, five, and 10 seconds after heel prick and the total length of cry were compared by Mann-Whitney U testing for the amethocaine and placebo groups.

\section{Results}

There were no significant differences in the characteristics of the infants in the two groups (table 1). The coefficient of reliability between the two assessors was $0.84,0.87,0.91,0.88$, and 0.94 for eye squeeze, brow bulge, deepened nasolabial folds, open mouth, and cry respectively. One infant (in the placebo group) was excluded from analysis because the lance was fired before the recording had started. In the five seconds immediately before the heel prick, the median cumulative NFCS scores were not significantly different in the two treatment groups (amethocaine 0 , interquartile range (IQR) 0-3 $v$ placebo 1 , IQR 0-4).

On the basis of the three second NFCS score, 20 of $30(67 \%)$ amethocaine treated infants showed no or minimal pain in response to the heel prick compared with 13 of $29(45 \%)$ in the placebo group $(\mathrm{p}=0.12)$ (table 2$)$. The median cumulative NFCS score over the three seconds after firing the lance was 3 (IQR 0-6) in the amethocaine group compared with 5

Table 2 Results for pain $v$ no or minimal pain and cry $v$ no cry for the amethocaine and placebo groups

\begin{tabular}{lll}
\hline & Amethocaine & Placebo \\
\hline No or minimal pain & $20(67)$ & $13(45)$ \\
Pain & $10(33)$ & $16(55)$ \\
& & $\mathrm{p}=0.12$ \\
Cry & $20(67)$ & $13(45)$ \\
No cry & $10(33)$ & $16(55)$ \\
& & $\mathrm{p}=0.12$
\end{tabular}

Values in parentheses are percentages. 


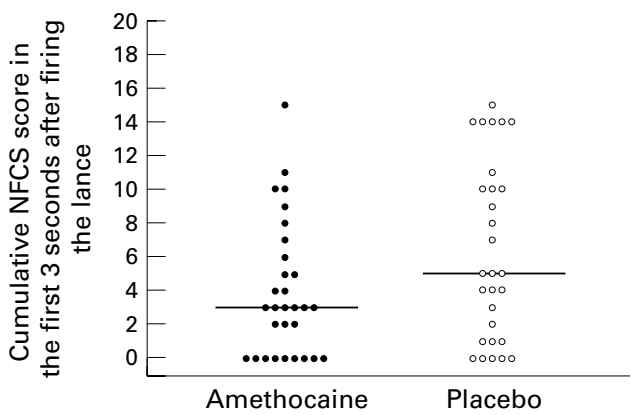

Figure 1 Cumulative neonatal facial coding system (NFCS) score in the first three seconds after the lance was fired. Horizontal bars indicate the median value in each group. The difference between the groups is not significant.

(IQR 1-10) in the placebo group ( $\mathrm{p}=0.07)$ (fig 1). Twenty of $30(67 \%)$ in the amethocaine treated group compared with 13 of $29(45 \%)$ in the placebo treated group did not cry at all in the five seconds immediately after firing of the lance $(p=0.12)$ (table 2). Logistic regression indicated that neither the response to heel prick nor the effect of amethocaine were influenced by the following: the cumulative NFCS score over five seconds before heel prick, birth weight, and gestational age.

The median cumulative NFCS score at five seconds (amethocaine 8, IQR 4-15 v placebo 15, IQR 7-20; $\mathrm{p}=0.06)$ and ten seconds (amethocaine 14, IQR 9-38 $v$ placebo 28, IQR $4-39, \mathrm{p}=0.13$ ) after heel prick were less in the amethocaine group but not statistically different. In those infants who cried, the median total length of cry was nine seconds (IQR $0-72)$ in the amethocaine group $(n=10)$ compared with seven seconds (IQR $0-64$ ) in the placebo treated group $(n=16) \quad(p=0.3)$. Twenty two of $30(73 \%)$ samples were collected at the first attempt in the amethocaine group and 22 of $29(76 \%)$ in the placebo group. No local skin reactions were seen after application of amethocaine or placebo.

\section{Discussion}

Several studies have investigated the use of topical lignocaine or a eutectic mixture of lignocaine/prilocaine (EMLA) for pain relief during HPBS. A small but statistically significant reduction in visual analogue scores was found after heel prick in infants treated with topical lignocaine, with no influence on the increase in heart rate. ${ }^{15}$ Rushforth et $a l^{16}$ and Larsson $e t a l^{18}$ used the NFCS to assess the effect of lignocaine and EMLA respectively on the response to heel prick with manual lances in randomised placebo controlled trials and could show no local anaesthetic effect. In an open study, the use of a spring loaded lance alone was shown to be less painful than the use of EMLA with a manual lance in terms of heart rate variability, respiratory rate, and transcutaneous oxygen and carbon dioxide tensions. ${ }^{17}$

Amethocaine as a $4 \%(\mathrm{w} / \mathrm{w})$ gel (Ametop) has been developed for use as a topical local anaesthetic by McCafferty $e t a l^{27}$ and is licensed for use in term infants over 1 month of age. Structurally it has a lipophilic benzene ring attached to a tertiary amine group by an ester link chain, differing from local anaesthetics like lignocaine and prilocaine which have an amide link chain. It is rapidly metabolised in the blood by pseudocholinesterase. Transient erythema is common because of its vasodilator effect, in contrast with the pallor of vasoconstriction produced by EMLA. We have explored the effectiveness of amethocaine gel in the newborn using graded stimuli to elicit the cutaneous withdrawal reflex. ${ }^{22}{ }^{23}$ It has a local anaesthetic action on the dorsum of the foot, which can be detected 30 minutes after application and which lasts for two to five hours. It is extremely effective in reducing the pain of venepuncture in both term and preterm infants. ${ }^{24}$ In a recent study using a similar protocol to this, we showed that $84 \%$ of amethocaine treated infants showed no or minimal pain to the needle insertion compared with $30 \%$ of placebo treated infants, a highly significant difference. ${ }^{24}$

In this study, infants treated with topical amethocaine gel showed a lesser pain response to the heel prick by the parameters measured but in no case were the differences between the treatment and the placebo group statistically significant. As the difference between the treatment and the placebo group was seen within each of the NFCS pain parameters, we think the difference is likely to be a real effect of the drug rather than a chance one. It seems therefore that amethocaine gel only has a mild effect on the pain of heel prick when compared with placebo, in contrast with its pronounced effect on the pain of venepuncture. A larger sample size may have shown a statistically but not clinically significant effect; a topical local anaesthetic must be effective in almost every patient to be of any use in real life. The scoring system we used to assess procedural pain has been previously validated and was identical with the method used in our venepuncture study. ${ }^{24}$ There was good agreement between the tape assessors for each of the five characterisitics measured.

What are the possible reasons for this apparent failure of topical amethocaine gel to relieve the pain of HPBS in the newborn infant? Firstly, we used an automated spring loaded lancing device, a method of blood sampling that is very popular with diabetic children who use it repeatedly with very little pain. Such a device has been shown to be less painful for HPBS in the newborn when compared with a manual lance..$^{172}{ }^{29}$ If we had studied the effect of amethocaine on HPBS by the more painful manual lance, we may have found a significant anaesthetic action. Against this explanation is the fact that amethocaine has a pronounced effect on the pain of venepuncture, itself a less painful procedure in the newborn than a manual heel prick. ${ }^{67}$ Secondly a heel prick is different from a venepuncture in that some squeezing of the heel is necessary to obtain the blood. This in itself is painful (or at least it elicits the behavioural response from the infant that we are measuring as pain). We deliberately chose to make our study a pragmatic one, studying heel pricks as they are actually carried 
out, by different personnel in different ways, to see if amethocaine reduced or abolished the pain of the heel prick under field conditions. Our protocol did not include a period immediately after firing the lance during which no squeezing of the heel was permitted. If squeezing were routinely performed in the first three seconds after lancing, this would have masked any beneficial effect of the amethocaine gel. In practice, however, there was little if any heel squeezing in this period (which is why we chose the pain response in these three seconds as a primary outcome measure). Thirdly, the skin of the heel is different from that on the dorsum of the hand or foot. The epidermis of the heel is considerably thicker, and the perfusion of the skin of the heel is two to three times that of the dorsum of the hand. ${ }^{30}$ This may result in decreased absorption, increased metabolism, and increased removal of amethocaine with diminution of any local anaesthetic effect.

In conclusion, topical amethocaine gel does not have a clinically important effect on the pain of heel prick blood sampling and its use for this purpose cannot therefore be recommended. Alternative approaches to the relief of pain from this procedure should be explored.

This study was funded entirely by the Higher Education Funding Council. We are grateful to Dr Dermot McCafferty of Queen's University Belfast who supplied the placebo gel and to Sarah Pacey and Sarah Charlesworth of Nottingham City Hospital Pharmacy who packaged the gels and carried out the randomisation and coding.

1 Barker DP, Rutter N. Exposure to invasive procedures in neonatal intensive care unit admissions. Arch Dis Child Fetal Neonatal Ed 1995;72:F47-8.

2 Johnston CC, et al. A cross-sectional survey of pain and pharmacological analgesia in Canadian neonatal intensive care units. Clinical fournal of Pain 1997;13:308-12.

3 McGraw MB. Neural maturation as exemplified in the changing reactions of the infant to pin prick. Child Der 1941;12:31-42.

4 Rich EC, Marshall RE, Volpe JJ. The normal neonata response to pin-prick. Dev Med Child Neurol 1974;16:432-4.

5 Owens ME, Todt EH. Pain in infancy: neonatal reaction to a heel lance. Pain 1984;20:77-86.

6 Shah VS, et al. Neonatal pain response to heel stick vs venepuncture for routine blood sampling. Arch Dis Child Fetal Neonatal Ed 1997;77:F143-4.

7 Larsson BA, et al. Venipuncture is more effective and less painful than heel lancing for blood tests in neonates. Pedi atrics 1998;101:882-6.

8 Fitzgerald M, Millard C, McIntosh N. Cutaneous hypersensitivity following peripheral tissue damage in newborn infants and its reversal with topical anaesthesia. Pain $1989 ; 39 \cdot 31-6$.

9 Grunau RV, Whitfield MF, Petrie JH. Pain sensitivity and temperament in extremely low-birth-weight premature toddlers and preterm and full-term controls. Pain 1994;58:341-6.

10 Taddio A, et al. Effect of neonatal circumcision on pain responses during vaccination in boys [see comments]. Lancet 1995;345:291-2.

11 Taddio A, et al. Effect of neonatal circumcision on pain response during subsequent routine vaccination /see comments]. Lancet 1997;349:599-603.

12 Porter FL, Grunau RE, Anand KJ. Long-term effects of pain in infants. $\mathcal{F}$ Dev Behav Pediatr 1999;20:253-61.

13 Oberlander TF, et al. Biobehavioral responses to acute pain in adolescents with a significant neurologic impairment. Clinical fournal of Pain 1999;15:201-9.

14 Barker DP, Rutter N. Lignocaine ointment and local anaesthesia in preterm infants. Arch Dis Child Fetal Neonatal Ed 1995;72:F203-4

15 Wester U. Analgesic effect of lidocaine ointment on intact skin in neonates. Acta Paediatr 1993;82:791.

16 Rushforth JA, et al. Can topical lignocaine reduce behavioural response to heel prick? Arch Dis Child Fetal Neonatal Ed 1995;72:F49-51.

17 McIntosh N, van Veen L, Brameyer H. Alleviation of the pain of heel prick in preterm infants. Arch Dis Child Fetal Neonatal Ed 1994;70:F177-81.

18 Larsson BA, et al. Does a local anaesthetic cream (EMLA) alleviate pain from heel-lancing in neonates? Acta alleviate pain from heel-lancing

19 Woolfson AD, McCafferty DF, Boston V. Clinical experiences with a novel percutaneous amethocaine preparation: prevention of pain due to venepuncture in children. $\mathrm{Br} F$ Clin Pharmacol 1990;30:273-9.

20 Doyle E, et al. An evaluation of a new self-adhesive patch preparation of amethocaine for topical anaesthesia prior to venous cannulation in children. Anaesthesia 1993;48:1050-2.

21 Lawson RA, et al. Evaluation of an amethocaine gel preparation for percutaneous analgesia before venous cannulation in children. Br $\mathcal{F}$ Anaesth 1995;75:282-5.

22 Jain A, Rutter N. Local anaesthetic effect of topical amethocaine gel in neonates: randomised controlled trial. Arch Dis Child Fetal Neonatal Ed 2000;82:F42-5.

23 Jain A, Rutter N. Topical amethocaine gel inthe newborn infant: how soon does it work and how long does it last? Early Hum Dev 1999.

24 Jain A, Rutter N. Does topical amethocaine gel reduce the pain of venepuncture in newborn infants? A randomised double blind controlled trial. Arch Dis Child Fetal Neonatal Ed 2000;83:211-4.

25 Rushforth JA, Levene MI. Behavioural response to pain in healthy neonates. Arch Dis Child Fetal Neonatal Ed 1994;70:F174-6

26 Craig KD, et al. Pain in the preterm neonate: behavioural and physiological indices [published erratum appears in Pain 1993;54:111]. Pain 1993;52:287-99.

27 McCafferty DF, Woolfson AD, Boston V. In vivo assessment of percutaneous local anaesthetic preparations. $\mathrm{Br} F$ Anaesth 1989;62:17-21.

28 Harpin VA, Rutter N. Making heel pricks less painful. Arch Dis Child 1983;58:226-8.

29 Barker DP, Latty BW, Rutter N. Heel blood sampling in preterm infants: which technique? Arch Dis Child Fetal Neonatal Ed 1994;71:F206-8.

30 Larsson BA, et al. Regional variations in skin perfusion and skin thickness may contribute to varying efficacy of topical, local anaesthetics in neonates. Paediatr Anaesth $1996 ; 6: 107-10$ 